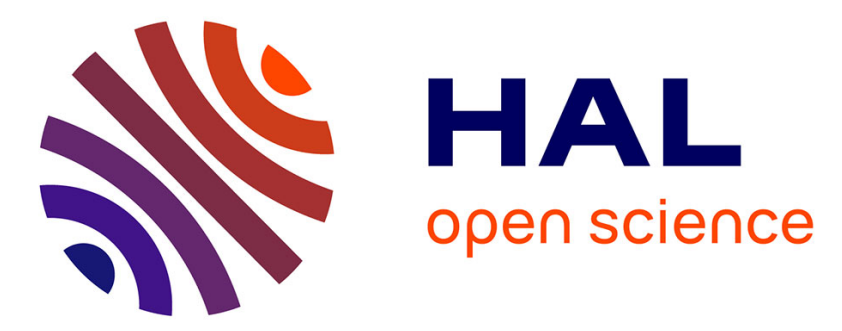

\title{
Parametric study of wave propagation in hierarchical auxetic perforated metamaterials
}

Kévin Billon, Morvan Ouisse, Emeline Sadoulet, Fabrizio Scarpa, Manuel Collet

\section{To cite this version:}

Kévin Billon, Morvan Ouisse, Emeline Sadoulet, Fabrizio Scarpa, Manuel Collet. Parametric study of wave propagation in hierarchical auxetic perforated metamaterials. SPIE Active and Passive Smart Structures and Integrated Systems, Mar 2016, Las Vegas, United States. 10.1117/12.2221895 • hal03427078

\section{HAL Id: hal-03427078 \\ https://hal.science/hal-03427078}

Submitted on 12 Nov 2021

HAL is a multi-disciplinary open access archive for the deposit and dissemination of scientific research documents, whether they are published or not. The documents may come from teaching and research institutions in France or abroad, or from public or private research centers.
L'archive ouverte pluridisciplinaire HAL, est destinée au dépôt et à la diffusion de documents scientifiques de niveau recherche, publiés ou non, émanant des établissements d'enseignement et de recherche français ou étrangers, des laboratoires publics ou privés.

\section{다)(1) $(5$}

Distributed under a Creative Commons Attribution - NonCommerciall 4.0 International 


\title{
Parametric Study of Wave Propagation in Hierarchical Auxetic Perforated Metamaterials
}

\author{
K. Billon ${ }^{\mathrm{a}}$, M. Ouisse ${ }^{\mathrm{a}}$, E. Sadoulet-Reboul ${ }^{\mathrm{a}}$, F. Scarpa ${ }^{\mathrm{b}}$, and M. Collet $^{\mathrm{c}}$ \\ ${ }^{a}$ Applied Mechanics department, Femto-st Institute, 24 rue de l'Epitaphe, 25000 Besanon, \\ France \\ ${ }^{\mathrm{b}}$ Advanced Composites Centre for Innovation and Science (ACCIS), University of Bristol, BS8 \\ 1TR Bristol, United Kingdom \\ 'Tribology and System Dynamics Laboratorys (LTDS), 36 avenue Guy de Collongue, 69134 \\ Ecully, France
}

\begin{abstract}
The understanding of wave propagation in a metamaterial with hierarchical, auxetic rectangular perforations is presented in this work. The metamaterial is a 2D structure with chaining horizontal and vertical perforations exhibing auxetic in-plane behaviour. The unit cell of this lattice is identified as the reference level 0. Hierarchical structures are composed of structural elements which themselves have structure. At level 0, 4 rigid squares are present in the unit cell. In each square, the reference structure is used by applying a scale ratio to obtain the level 1. The same strategy is used to reach the upper level in each subunit. A geometric parametric investigation of these rectangular perforations using a numerical asymptotic homogenisation finite element approach is done. Some numerical eigenvalue tools are used for the dispersion analysis of this structure. It is first observed that the total width of Band gaps increases with the hierarchy. The porosity induced by the perforations is taken into account in the mechanical properties. The symmetry of the geometry in the $\mathrm{x}$-y plane allow to define the entire geometry of the unit cell using only 2 parameters: the void aspect ratio, the intercell spacing and the hierarchy level. When decreasing the intercell spacing, the total width of Band gaps increases and the effective stiffness in $\mathrm{x}$ and $\mathrm{y}$ directions decrease, allowing for increased rotations of the rigid sqares, so auxetic behaviour is greater. Hierarchical levels shift from isotropic to orthotropic, hierarchical levels are always auxetic.
\end{abstract}

Keywords: periodic, perforations, hierarchy, auxetic, mechanical properties, homogenisation.

\section{INTRODUCTION}

A periodic medium is a material or a structural system that exhibits spatial periodicity. The study of periodic structures has a long history in the field of vibrations and acoustics. This topic has interested researchers over the years, and a growing activity on this field is observed on the last years, with the objective of designing structures exhibiting properties that conventional ones cannot possess.

A very detailed review of historical origins, recent progress and future outlook of this topic has been published recently. ${ }^{1}$ The reader is invited to refer to this article and the following discussion ${ }^{2}$ that cover the most important aspects of this topic.

Dynamic performances of structured media at the macroscopic scale is of first interest for many engineering applications, with increasing number of papers either for passive ${ }^{3}$ or active ${ }^{4-7}$ devices.

Hierarchical structures are composed of structural elements witch themselves have structure. ${ }^{8-11}$

Auxetic materials are materials with a negative Poisson's ratio. For conventional materials, during an uniaxial tensile test, a reduction of the dimensions perpendicular of the load is observed, in the case of auxetic materials an increasing of these dimensions is obtained. ${ }^{12}$

Further author information: (Send correspondence to K. Billon)

K. Billon: E-mail: kevin.billon@femto-st.fr, Telephone: +33 (0)38166 6000

M. Ouisse: E-mail: morvan.ouisse@femto-st.fr, Telephone: +33 (0)3 81666046

Active and Passive Smart Structures and Integrated Systems 2016, edited by Gyuhae Park, Proc. of SPIE Vol. 9799, $979906 \cdot$ C 2016 SPIE · CCC code: 0277-786X/16/\$18 · doi: 10.1117/12.2221895 
Dispersion analysis of plates with hierarchical, auxetic rectangular perforations are described in this paper. A geometric parametric investigation of these rectangular perforations using a numerical asymptotic homogenisation finite element approach is done in order to understand the macroscopic behavior of the structured medium. This approach is valid for static and low frequency response of the structure of interest. Then, for the higher frequency range, a dispersion analysis of the system is investigated, allowing description of the wave propagation characteristics of the hierarchical system.

\section{GEOMETRY OF THE HIERARCHICAL PERFORATED AUXETIC LATTICE}

Figure 1a shows the structural dimensions of the square lattice with rectangular perforations. The symmetry of the geometry in the $x-y$ plane allow to define the entire geometry of the unit cell using only 2 parameters: the void aspect ratio, $A R=a / b$ and the intercell spacing $S .{ }^{13}$

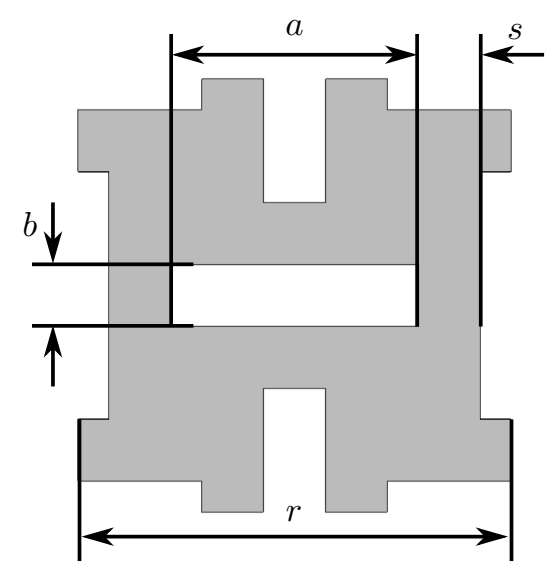

(a)

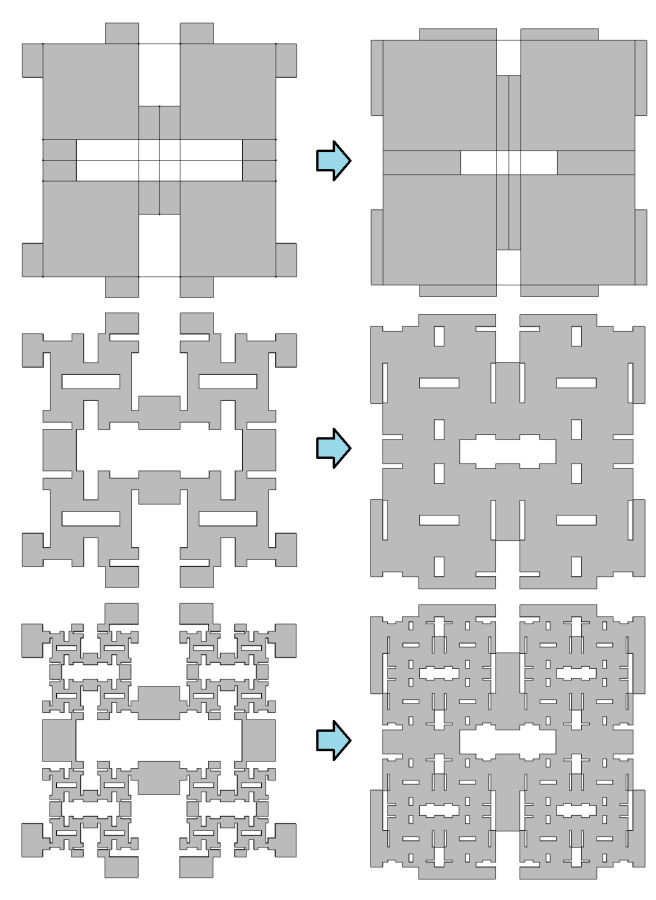

(b)

Figure 1: (a) Geometry parameters of the base unit cell. (b) Hierarchical, auxetic rectangular perforations at Levels 1,2 and 3 with $A R=4$ and from $S=0.2$ to $S=0.8$.

As a reference, the level $1^{13}$ is compared with the hierarchical levels 2 and 3 . Hierarchical structures are systems witch are composed of structural elements witch themselves have structure. At level 1, 4 rigid squares are present in the unitcell. In each square, the reference structure is used by applying a scale ratio to obtain the level 2. Exactly the same at level 3, in this subunit, there are 4 rigid squares, the reference structure is used by applying a second scale ratio to obtain the level 3 .

The parametric analysis is carried out with the aspect ratios $(A R)$, the intercell spacing $(S)$ and the level of hierarchy. Figure 1b shows how intercell spacing change in both level of hierarchy. Voids are larger than a low parameter $S$ and the porosity increases with the level of hierarchy.

\section{IN-PLANE MECHANICS}

The numerical asymptotic homogenisation is carried out with a commertial FE code. The unit cell shown in figure 1a is meshed using PLANE82 (8-nodes with 2 degrees of freedom) elements.

The homogenized stress-strain tensor $\bar{C}$ of the structure is given by 


$$
\bar{C}=\left(\begin{array}{ccc}
C_{11} & C_{12} & 0 \\
C_{21} & C_{22} & 0 \\
0 & 0 & C_{66}
\end{array}\right) .
$$

The engineering material parameters can be identified from the compliance matrix $\bar{S}$ linked to the stress-strain tensor $\bar{C}$ in the case of special orthotropic materials in plane strain, namely

$$
\bar{S}=\bar{C}^{-1}=\left(\begin{array}{ccc}
1 / E_{1} & -\nu_{21} / E_{2} & 0 \\
-\nu_{12} / E_{1} & 1 / E_{2} & 0 \\
0 & 0 & 1 / G_{12}
\end{array}\right) .
$$

3 virtual experiments are used to determine 2 Young's modulus $\left(E_{1}, E_{2}\right), 2$ Poisson's ratio $\left(\nu_{12}, \nu_{21}\right)$ and the shear modulus $\left(G_{12}\right)$. The reaction force and the displacement are then computed using the finite element model. For example, in the $\mathrm{x}$ tensile virtual test, the first one is used for effective elastic modulus calculation $E_{1}=\sigma_{1} / \varepsilon_{1}$ and the second is used for the Poisson's ratio ${ }^{14} \nu_{12}=-\varepsilon_{2} / \varepsilon_{1}$.

The results are normalised against the first mode of a plate with same overall dimensions. The stiffness and the density calculated as $E^{\prime}=E \times \phi^{2}$ and $\rho^{\prime}=\rho \times \phi$ with the porosity $\phi=V_{\text {void }} / V_{\text {tot }}$. The evolution of the porosity for various values of the intercell spacing $S$ at Levels 1, 2 and 3 is given in figure 2 a. The frequencies used for normalisation are presented in figure $2 \mathrm{~b}$. The normalised frequency band of interest is from 0 to 5 .

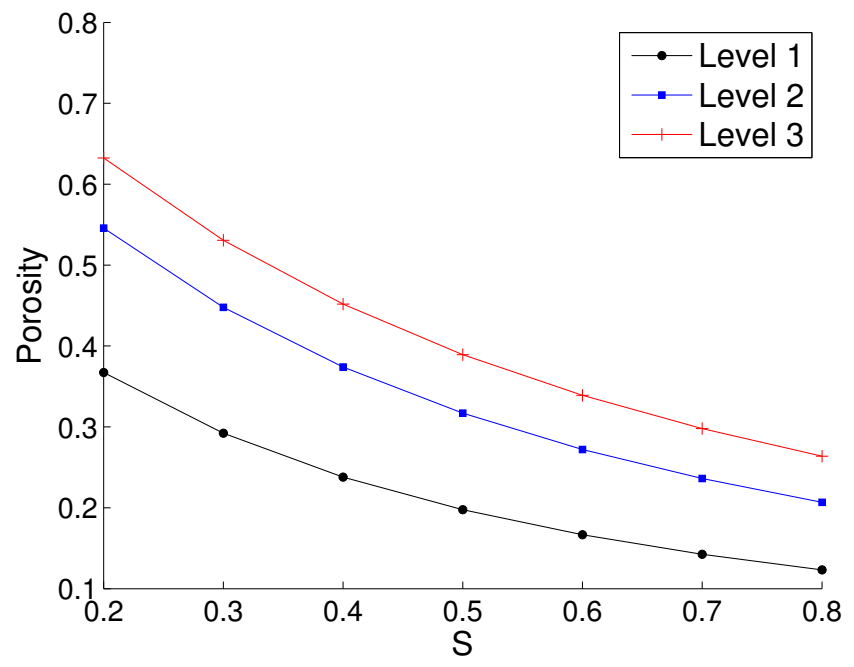

(a)

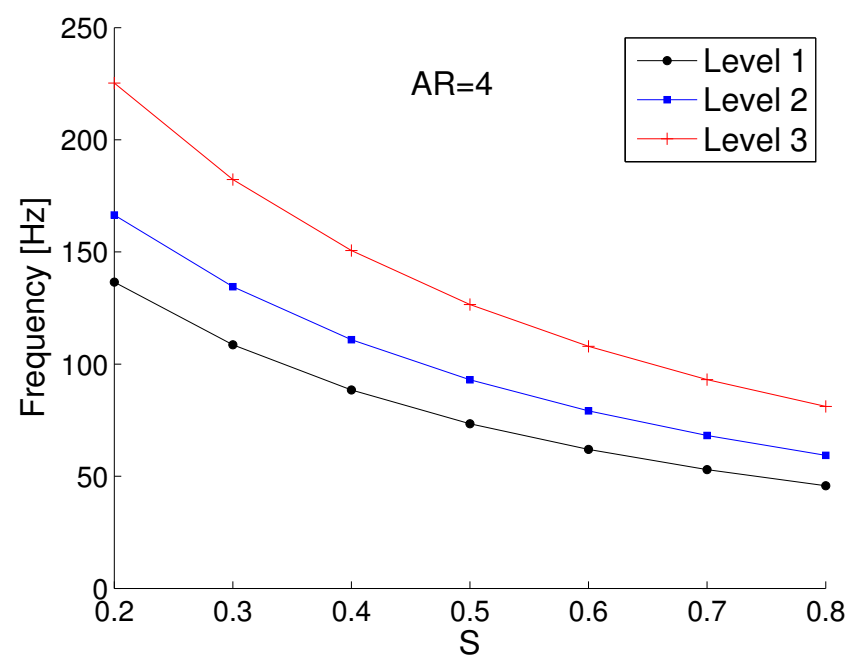

(b)

Figure 2: (a). Equivalent porosity for various sizes of the intercell spacing $S$ at Level 1, 2 and 3. (b)Frequencies used to normalise against the first mode of a plate with same overall dimensions including the porosity in various size of the intercell spacing $S$ at Level 1, 2 and 3.

The engineering material parameters can be identified using the numerical asymptotic homogenisation. The structure is considered as orthotropic materials in plane strain with 5 mechanical parameters, Young's modulus in $x$ and $y$ directions $\left(E_{1}\right.$ and $\left.E_{2}\right)$, Poisson's ratios $\left(\nu_{12}\right.$ and $\left.\nu_{21}\right)$ and the shear modulus $\left(G_{12}\right)$.

Figures $3 \mathrm{a}$ to $3 \mathrm{~b}$ show the evolution of the non dimensional mechanical properties of the homogenized structure. Particular shapes corresponding to singular points and deformed shapes are also shown in these figures. As seen in figure 3b, the Poisson's ratio effect is linked to the rotation of the rigid squares. Poisson's ratio effect increases with higher hierarchical level.

For increasing the intercell spacing $(S)$, the effective stiffness in $x$ direction increase in figures 3a, same tendency in the $y$ direction, allowing for decreased rotations of the rigid squares, so auxetic behaviour is greater. 
At level 1, the structure is isotropic, with the increase of the $S$ dimension the Poisson's ratio tends to become positive. The stiffness ratio between each levels in $x$ and $y$ directions decreases when the intercell spacing increases. At Level 2 and 3, jumps in mechanical properties are observed due to transition of parts of the subunits from contact to noncontact between voids figures 3a. Hierarchical levels shift from isotropic to orthotropic, hierarchical levels are always auxetic figure $3 \mathrm{~b}$.

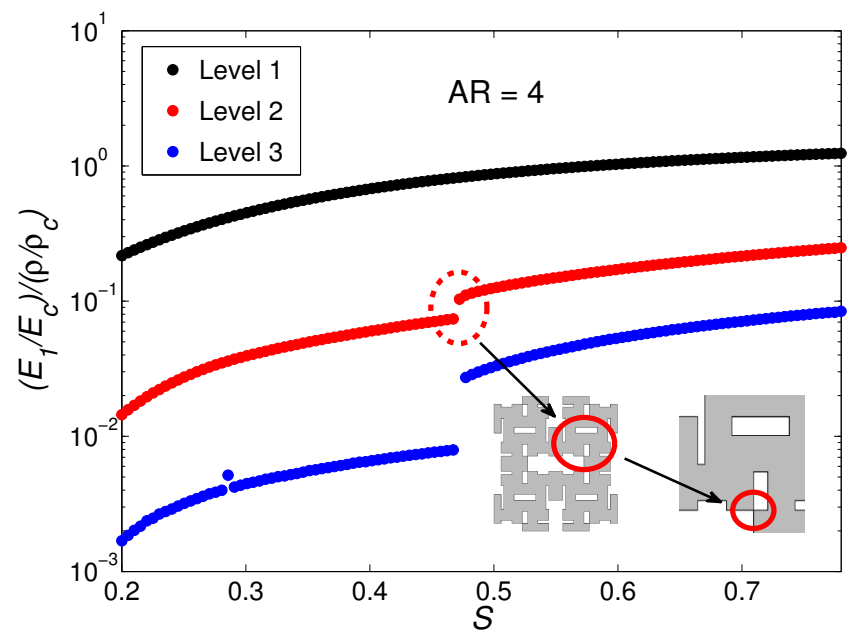

(a)

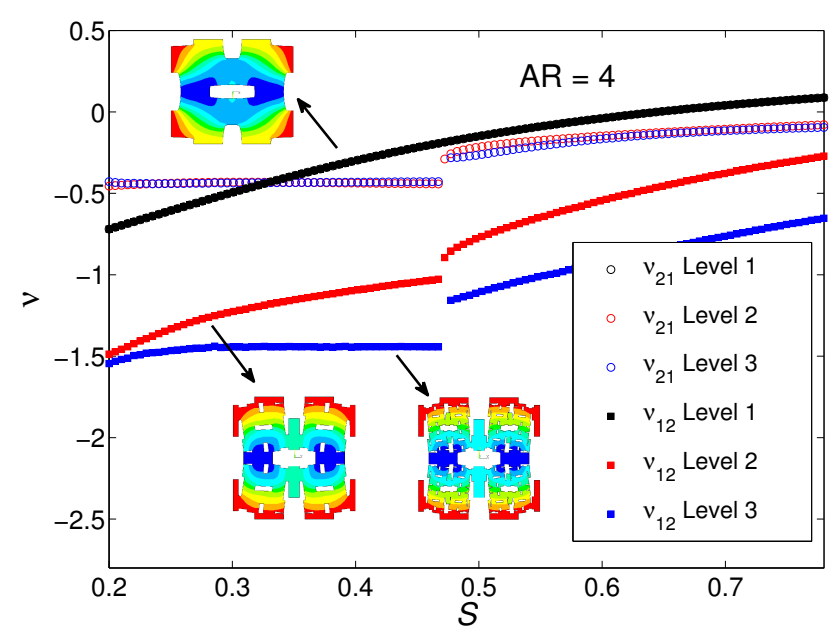

(b)

Figure 3: Variation of the nondimensional Youngs modulus $E_{1}$ (a) and in-plane Poisons ratios $\nu_{12}$ and $\nu_{21}$ (b) versus the intercell spacing $\mathrm{S}$ at Levels 1,2 and 3 for an aspect ratio of 4 .

\section{WAVE PROPAGATION}

The finite element simulations in order to obtain the dispersion curves are carried out with a commercial $\mathrm{FE}$ code.

The classical Floquet-Bloch approach is a method commonly used for the study of periodic structures. The material constitutive law is linear, elastic and isotropic. The real geometry is used. The periodicity is defined on the borders of the domain $u_{R}=e^{-j k_{x} r} u_{L}$ and $v_{R}=e^{-j k_{y} r} v_{L}$ where $u_{R}$ (resp. $v_{R}$ ) is the displacement on the right border and $u_{L}$ (resp. $v_{L}$ ) is the displacement on the left border in $x$ (resp. $y$ ) axis, $k_{x}$ and $k_{y}$ are respectively the wavenumbers in the $x$ and $y$ directions ${ }^{15}$ for details.

The harmonic homogeneous dynamical equilibrium of the system is driven by the following partial derivative problem

$$
\rho \omega^{2} u+\nabla \sigma=0, \sigma=C: \varepsilon,
$$

where $u \in \mathbb{R}^{3}$ is the displacement, $\sigma$ is the stress tensor, $C$ is the elastic tensor and $\epsilon$ is the strain tensor.

A parametric eigenvalue analysis is performed using the Pardiso solver, ${ }^{16}$ two parameters (wavenumbers) are considered, namely $k_{x} \in[0 \pi / r]$ and $k_{y} \in[0 \pi / r]$. The wave's dispersion curves of the undamped system are plotted on the contours of the first Brillouin zone. The frequencies defining the bandgaps can always be found by considering only the contour of the irreducible Brillouin zone for regular system. ${ }^{17}$ It is worth noticing that this method is extensively used in open literature, although no formal proof of its validity is given, and therefore the results obtained need to be looked at carefully. ${ }^{18}$

The results of the analysis correspond to dispersion diagrams which are determined for the various levels of hierarchy and different intercell spaces $(S)$. They provide the value of the wave numbers for varying angle of propagation through the lattice together with the corresponding eigenfrequencies. 
The band diagram onlu provides information on the contour of the Brillouin zone allowing identification of the bandgaps. Hence, only specific directions $\left(0^{\circ}, 45^{\circ}\right.$ and $\left.90^{\circ}\right)$ are investigated.

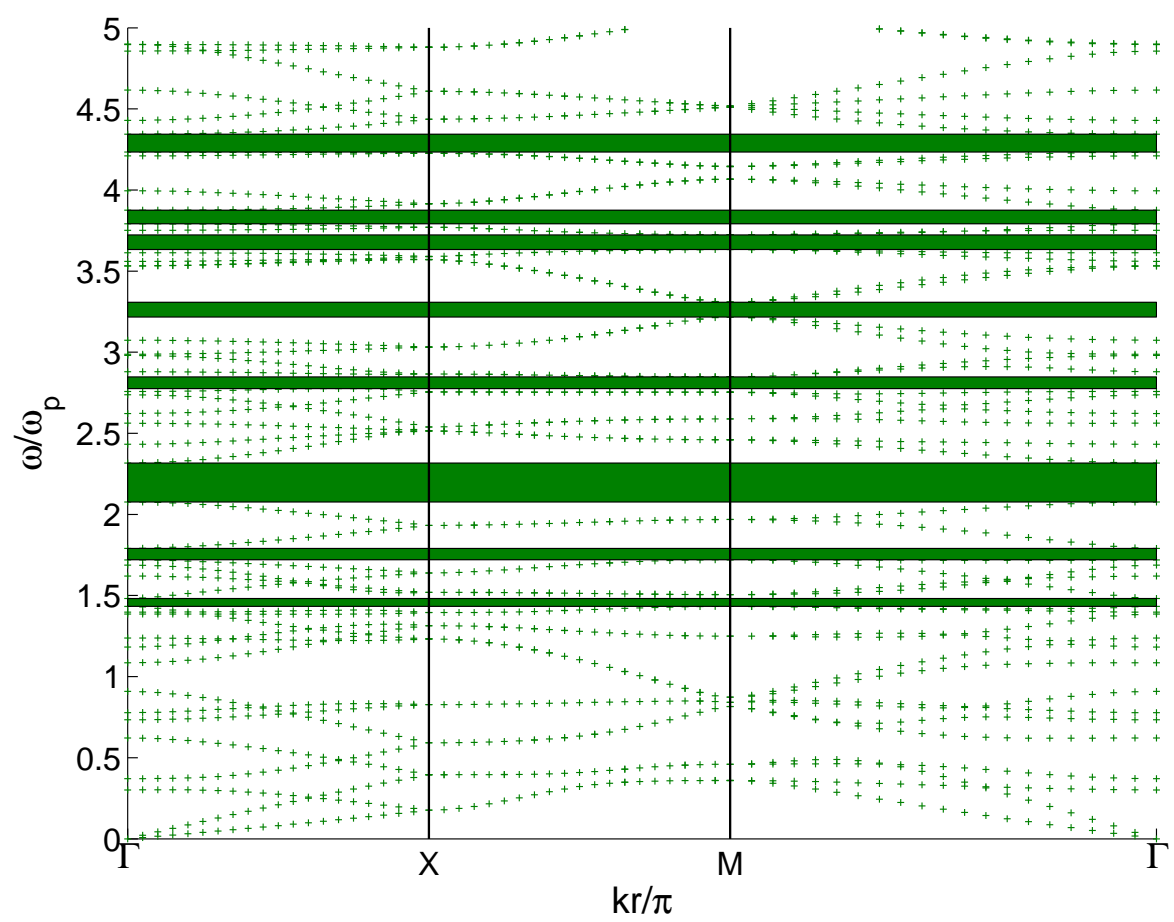

Figure 4: Dispersions in the k-space for the lattice with $A R=4, S=0.3$ for Level 2. Full coloured areas correspond to band gaps

Band gaps are observed at some specific values of $A R$ and $S$ (see 4). These band gaps are called omnidirectional band gap because whatever the direction of the wave propagation, this wave can not propagate. And there are also directional band gaps mean that for example in a frequency band waves are able to propagate along several directions greater or lesser extent. But in our case, a particular interest is given only in omnidirectional band gaps. The modal density increase with the hierarchy, it is true whatever the value of the parameter $S$ is.

This can clearly be seen in figure 5 that provide a synthetic view of the band gaps for the various geometry configurations.

\section{CONCLUSION}

Periodic, auxetic and hierarchical structures are interesting for waves propagation as they may exhibit complete or at least partial frequency bandgaps such that the associated waves cannot propagate through the structure. The determination of the dispersion curves is thus necessary to design specific structures for an absorption purpose. It is first observed that the total width of Band gaps increases with the hierarchy. When decreasing the intercell spacing, the total width of Band gaps increases and the effective stiffness in $\mathrm{x}$ and y directions decrease, allowing for increased rotations of the rigid sqares, so auxetic behaviour is greater. Hierarchical levels shift from isotropic to orthotropic, hierarchical levels are always auxetic. Other geometry or perforation can be investigate.

\section{ACKNOWLEDGMENTS}

This work was financed by The French National Research Agency under grant number ANR-12-JS09-008-COVIA. It has been performed in cooperation with the Labex ACTION program (ANR-11-LABX-0001-01). The Authors also acknowledge the support of the FP7-AAT-2012-RTD-L0-341509 MorphElle project for providing part of the computational tools used in this work. 


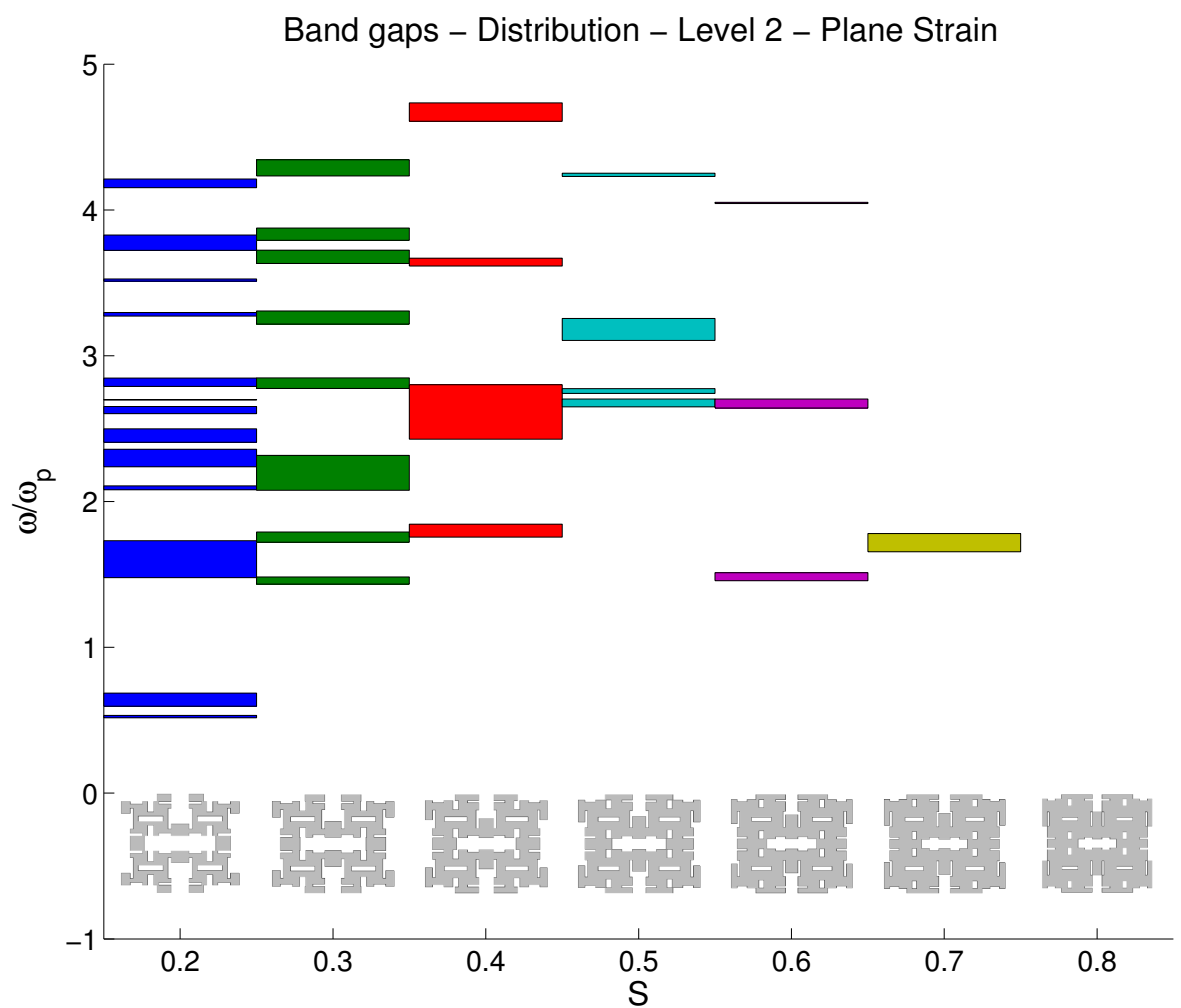

Figure 5: Band gap distribution versus different $S$-parameter configurations for Level 2.

\section{REFERENCES}

[1] M. Hussein, M. Leamy, and M. Ruzzene, "Dynamics of phononic materials and structures: Historical origins, recent progress, and future outlook," Applied Mechanics Reviews 66(4), p. 040802, 2014.

[2] B. Mace, "Discussion of 'Dynamics of phononic materials and structures: Historical origins, recent progress, and future outlook '(M.I. Hussein, M.J. Leamy and M. Ruzzene, Applied Mechanics Reviews, 66(4):040802, 2014)," Applied Mechanics Reviews 66(4), p. 045502, 2014.

[3] F. Scarpa, M. Ouisse, M. Collet, and K. Saito, "Kirigami auxetic pyramidal core: mechanical properties and wave propagation analysis in damped lattice," Journal of Vibration and Acoustics 135(4), p. 041001, 2013.

[4] M. Collet, M. Ouisse, and F. Tateo, "Adaptive metacomposites for vibroacoustic control applications," Sensors Journal, IEEE 14(7), pp. 2145-2152, 2014.

[5] M. Collet, M. Ouisse, M. Ichchou, and R. Ohayon, "Semi-active optimization of $2 \mathrm{~d}$ waves dispersion into shunted piezocomposite systems for controlling acoustic interaction," in ASME 2011 Conference on Smart Materials, Adaptive Structures and Intelligent Systems, pp. 79-87, American Society of Mechanical Engineers, 2011.

[6] Z. Huang and C. Su, "Band gaps of a two-dimensional periodic graphenelike structure," Journal of Vibration and Acoustics 135(4), p. 041002, 2013.

[7] F. Tateo, M. Collet, M. Ouisse, M. Ichchou, K. Cunefare, and P. Abbe, "Experimental characterization of a bi-dimensional array of negative capacitance piezo-patches for vibroacoustic control," Journal of Intelligent Material Systems and Structures, p. 1045389X14536006, 2014.

[8] R. Norris, J. Hamel, and P. Nadeau, "Phononic band gap crystals with periodic fractal inclusions: Theoretical study using numerical analysis," Journal of Applied Physics 103(10), p. 104908, 2008. 
[9] S. Castiñeira-Ibáñez, C. Rubio, J. Redondo, and J. Sánchez-Pérez, "Quantitative characterization of bandgap properties of sets of isolated acoustic scatterers arranged using fractal geometries," Applied Physics Express 7(4), p. 042201, 2014.

[10] Y. Tang, G. Lin, L. Han, S. Qiu, S. Yang, and J. Yin, "Design of hierarchically cut hinges for highly stretchable and reconfigurable metamaterials with enhanced strength," Advanced Materials 27(44), pp. 7181-7190, 2015.

[11] Q. Lim, P. Wang, S. Koh, E. Khoo, and K. Bertoldi, "Wave propagation in fractal-inspired self-similar beam lattices," Applied Physics Letters 107(22), p. 221911, 2015.

[12] M. Ruzzene and F. Scarpa, "Directional and band-gap behavior of periodic auxetic lattices," physica status solidi (b) 242(3), pp. 665-680, 2005.

[13] A. Slann, W. White, F. Scarpa, K. Boda, and I. Farrow, "Cellular plates with auxetic rectangular perforations," Physica status solidi (b) 252(7), pp. 1533-1539, 2015.

[14] V. Salit and T. Weller, "On the feasibility of introducing auxetic behavior into thin-walled structures," Acta Materialia 57(1), pp. 125-135, 2009.

[15] M. Collet, M. Ouisse, M. Ruzzene, and M. Ichchou, "Floquet-bloch decomposition for the computation of dispersion of two-dimensional periodic, damped mechanical systems," International Journal of Solids and Structures 48(20), pp. 2837-2848, 2011.

[16] O. Schenk and K. Gärtner, "Solving unsymmetric sparse systems of linear equations with pardiso," Future Generation Computer Systems 20(3), pp. 475-487, 2004.

[17] J. Joannopoulos, S. Johnson, J. Winn, and R. Meade, Photonic crystals: molding the flow of light, Princeton university press, 2011.

[18] R. Craster, T. Antonakakis, M. Makwana, and S. Guenneau, "Dangers of using the edges of the brillouin zone," Physical Review B 86(11), p. 115130, 2012. 